Tema: Metalurgia Secundária

\title{
ANÁLISE DE INCLUSÕES NÃO METÁLICAS EM AÇO POR ESPECTROMETRIA DE EMISSÃO ÓPTICA*
}

\author{
Maria Cecília Junqueira Murta ${ }^{1}$ \\ Rodrigo Camargos Barroso ${ }^{2}$ \\ Dagoberto Brandão Santos ${ }^{3}$
}

\section{Resumo}

A espectrometria de emissão óptica é uma metodologia de análise de amostras sólidas utilizada pelas indústrias no controle da composição química de seus materiais. Nesta técnica, uma centelha é direcionada à amostra a ser analisada provocando a excitação dos elétrons de seus elementos, seguida pela emissão de radiação que é convertida em concentração química. Um recente avanço obtido nesta área analítica foi o desenvolvimento do sistema PDA/OES (análise de discriminação de pulsos). Esta ferramenta complementa a espectrometria de emissão óptica, permitindo que ela forneça além da composição química da amostra, o seu conteúdo de inclusões não metálicas. Trata-se de um progresso na investigação de inclusões, que atualmente é desempenhada principalmente pelos métodos de microscopia óptica e eletrônica de varredura. O propósito deste trabalho foi desenvolver uma metodologia de análise de inclusões através da espectrometria de emissão óptica e comparar os resultados obtidos com as técnicas clássicas de microscopia para, assim, possibilitar sua utilização no controle de inclusões durante a fabricação do aço.

Palavras-chave: Inclusões; Espectrometria de emissão óptica; PDA/OES; SparkDAT.

\section{ANALYSIS OF NON-METALLIC INCLUSIONS IN STEEL BY OPTICAL EMISSION SPECTROMETRY}

\section{Abstract}

The optical emission spectrometry is an analysis methodology of solid samples used in industries to control the chemical composition of their materials. In this technique, a spark is directed to the sample causing the excitation of electrons from its elements and the subsequent radiation emission that is converted to chemical concentration. A recent advance achieved in this area was the development of the PDA/OES system (pulse discrimination analysis). This tool complements the optical emission spectrometry allowing the non-metallic inclusions analysis in steel. This is a progress in the inclusions investigation, which is currently performed by optical microscopy and scanning electron microscopy. The purpose of this study was to develop a methodology for inclusions analysis using the optical emission spectrometry and compare the results obtained with classical techniques of analysis, thus enabling the use of this system in inclusions control during steel manufacturing.

Keywords: Inclusions; Optical emission spectrometry; PDA/OES; Spark-DAT.

1 Engenheira Química, Gerência de Qualidade e Pesquisa, Siderúrgica da Vallourec Tubos do Brasil, Belo Horizonte, MG, Brasil.

2 Engenheiro Metalurgista, MSc, Gerência de Qualidade e Pesquisa, Siderúrgica da Vallourec Tubos do Brasil, Belo Horizonte, MG, Brasil.

3 Engenheiro Metalurgista, Dr., Professor, Departamento de Engenharia Metalúrgica da Universidade Federal de Minas Gerais, Belo Horizonte, MG, Brasil.

\footnotetext{
* Contribuição técnica ao 45 Seminário de Aciaria - Internacional, 25 a 28 de maio de 2014,
} 


\section{INTRODUÇÃO}

O aumento da competitividade no mercado siderúrgico tem levado as empresas a uma busca constante pela melhoria da qualidade de seus produtos. Diante deste cenário, um dos principais tópicos estudados no processo de fabricação de aço é o surgimento de inclusões não metálicas.

As inclusões não metálicas como a apresentada na Figura 1 são impurezas presentes nos aços que alteram suas propriedades em maior ou menor grau dependendo da quantidade, do tamanho, da morfologia e da composição química das mesmas. Em sua maioria podem ser consideradas deletérias ao produto. De acordo com Kiessling et al. [1], elas são originadas de reações durante o processo de fabricação, de precipitação durante o resfriamento ou ainda são resultantes de incorporação mecânica de materiais com os quais o aço líquido entra em contato.

Figura 1. Micrografia de uma inclusão examinada ao microscópio eletrônico de varredura.

As indústrias siderúrgicas têm procurado reduzir e controlar o nível de inclusões não metálicas nos aços no sentido de produzir "aços mais limpos" e, consequentemente, mais homogêneos e com melhores propriedades mecânicas.

Como auxílio no controle das inclusões presentes no aço estão disponíveis diversas técnicas de caracterização. Os métodos mais utilizados são a microscopia eletrônica de varredura (MEV) e a microscopia óptica (MO). Contudo, o longo tempo para a execução da análise e a experiência de uma preparação mais elaborada da amostra são fatores limites da aplicação dessas técnicas para a produção em linha.

Por causa disso, grandes avanços vêm sendo desenvolvidos para reduzir o tempo requerido para este tipo de análise. Um exemplo é o surgimento do sistema PDA/OES (análise de discriminação de pulsos) também designado por Spark-DAT (aquisição e tratamento de dados das centelhas) que, ao ser acoplado a um espectrômetro de emissão óptica, permite avaliar em poucos segundos o número, o tipo e o tamanho das inclusões presentes em uma amostra.

O Spark-DAT trata-se de um pacote composto por hardware, software e algoritmos e atua como técnica complementar a espectrometria óptica que fornece somente dados de composição química. Seu princípio de funcionamento se baseia na medida da intensidade da radiação emitida pelos átomos presentes em uma amostra após receberem energia proveniente de centelhas elétricas aplicadas em sua superfície.

De acordo com Li et al. [2], na tradicional análise de composição química, os valores de intensidade de radiação obtidos após o centelhamento são somados e transformados em concentração. $\mathrm{Na}$ avaliação de inclusões, contudo, as intensidades originadas por cada centelha são adquiridas separadamente e submetidas a um tratamento matemático especial. O resultado é visto em um diagrama de intensidades como o da Figura 2, onde o eixo y representa as

* Contribuição técnica ao 45 Seminário de Aciaria - Internacional, 25 a 28 de maio de 2014, 
intensidades mensuradas para cada centelha e o eixo x, o número de centelhas descarregadas.

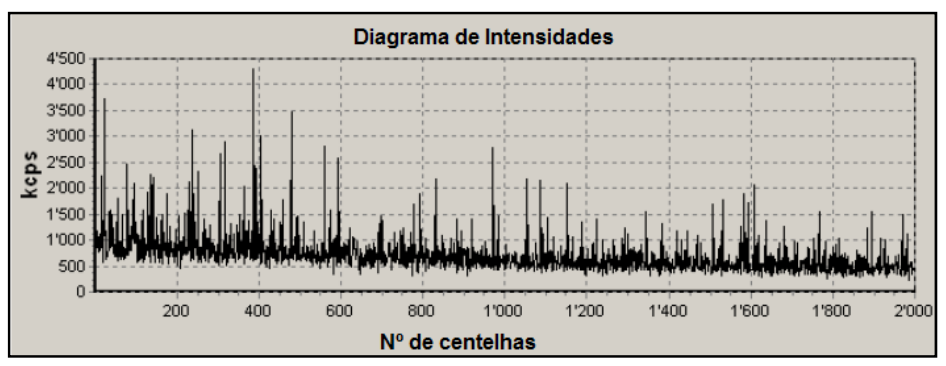

Figura 2. Diagrama de intensidades [3].

Os picos observados no diagrama de intensidades surgem quando uma centelha atinge uma inclusão. Isto acontece uma vez que, nesta região da amostra, o teor dos elementos presentes é maior do que a sua concentração na matriz. Segundo Li et al. [2], a intensidade dos picos exibidos em uma análise depende da quantidade de átomos contidos nas inclusões excitadas pelas centelhas. Assim, ela está relacionada com o tamanho da inclusão e com a concentração do elemento analisado na mesma. Já o número de picos encontrados está relacionado ao número de inclusões presentes.

Baseado neste princípio, o sistema Spark-DAT possibilita a avaliação do número de inclusões contidas em uma amostra através da contagem dos picos gerados por elemento químico. E, efetuando a correspondência dos picos que porventura sejam exibidos simultaneamente por diferentes elementos, permite obter a composição química das inclusões, conforme ilustrado pela Figura 3.

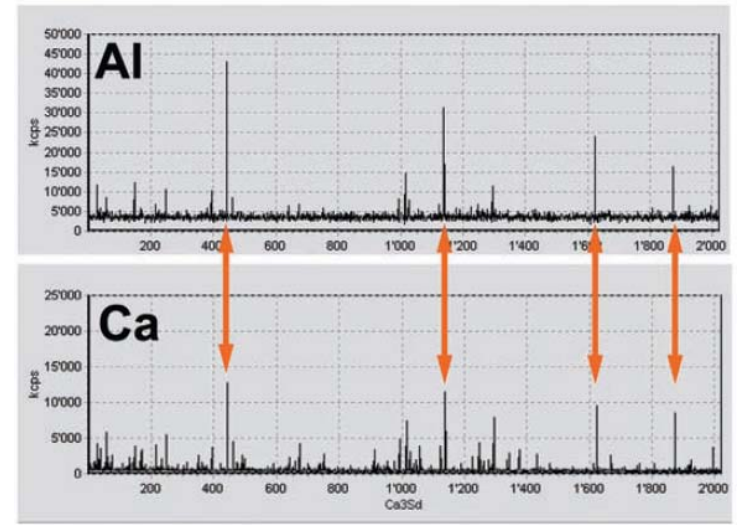

Figura 3. Determinação da composição química das inclusões [4].

Neste contexto, o presente trabalho teve como propósito desenvolver uma metodologia de análise de inclusões em amostras de aço utilizando como técnica de caracterização a espectrometria de emissão óptica associada ao Spark-DAT. Esta metodologia foi comparada com as técnicas clássicas de microscopia para avaliar sua possível utilização na identificação de inclusões durante as etapas de fabricação, permitindo intervenções imediatas que garantam a qualidade do produto final.

\section{MATERIAIS E MÉTODOS}

Para facilitar o entendimento, o estudo foi separado em três etapas. A primeira etapa consistiu na seleção e preparação de amostras de tubos de aço em que foram

* Contribuição técnica ao $45^{\circ}$ Seminário de Aciaria - Internacional, 25 a 28 de maio de 2014, 


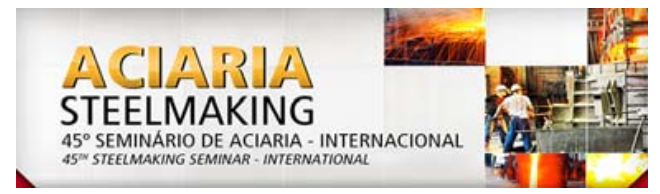

detectadas inclusões. Doze amostras $\left(A_{1}\right.$ a $\left.A_{12}\right)$ foram coletadas e preparadas conforme o procedimento descrito na norma ASTM E45 [5] (corte, lixamento e polimento). A Figura 4a mostra o resultado final desta etapa.

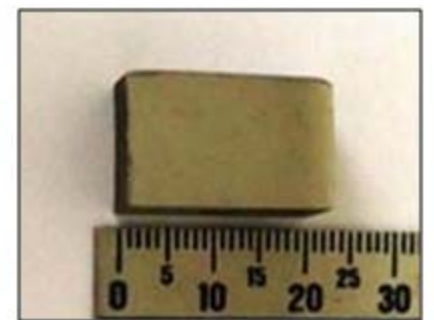

(a)

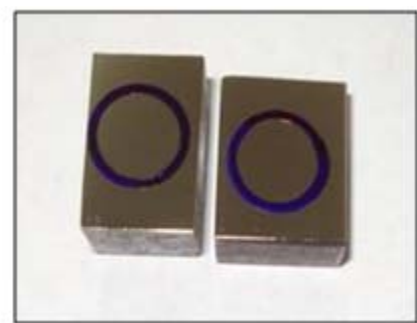

(b)

Figura 4. (a) Amostra preparada para análise por microscopia. (b) Região demarcada para análise por microscopia e Spark-DAT.

Em seguida, uma região circular de diâmetro de $10 \mathrm{~mm}$ foi marcada no centro da superfície polida de cada amostra como mostra a figura 4b. O objetivo desta marcação foi restringir a um mesmo local a área avaliada pelo MEV e Spark-DAT. Este tamanho corresponde à área atingida pelo centelhamento em uma análise por espectrometria óptica. Para a análise de microscopia óptica respeitou-se 0 procedimento descrito na norma ASTM E45 [5] método D que indica uma área mínima para análise de $160 \mathrm{~mm}^{2}$.

$\mathrm{Na}$ segunda etapa do trabalho, as amostras foram submetidas a ensaios no MEV (amostras $A_{1}$ a $A_{9}$ ) e no microscópio ótico (amostras $A_{10}$ a $A_{12}$ ) para a caracterização das inclusões encontradas. No microscópio eletrônico de varredura foram escolhidas aleatoriamente 30 inclusões para análise localizadas no interior da região delimitada em cada uma das amostras, conforme indicado na Figura 5. Para cada inclusão foi determinado o tamanho e a composição química.

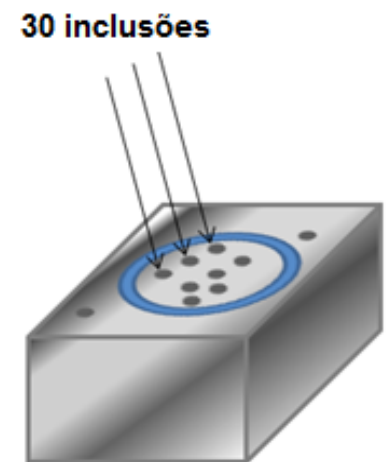

Figura 5. Seleção de inclusões para análise no microscópio eletrônico de varredura.

O equipamento utilizado foi o Microscópio Eletrônico de Varredura modelo Inspect S50 e marca $\mathrm{FEI}$, com um detector de raios-x por energia dispersiva (EDS) modelo Gêneses e marca EDAX, acoplado ao mesmo.

As demais amostras $\left(A_{10}\right.$ a $\left.A_{12}\right)$ foram examinadas no microscópio óptico modelo Metalloplan e marca Leitz para a caracterização segundo o Método D proposto na norma ASTM E45 [5]. Cada uma das inclusões encontradas foi classificada segundo os seguintes critérios:

- Tipo de Inclusão: Tipos A (Sulfetos), B (Alumina), C (Silicatos) e D (Óxidos).

- Espessura: séries grossa ou fina.

- Severidade: 6 níveis variando de 0,5 a 3,0, segundo a quantidade de inclusões por unidade de superfície.

* Contribuição técnica ao $45^{\circ}$ Seminário de Aciaria - Internacional, 25 a 28 de maio de 2014, 
Uma vez identificados os elementos componentes das inclusões avaliadas pela microscopia, o último passo deste estudo foi o de desenvolver um programa de análise no espectrômetro de emissão óptica modelo ARL 4460 da marca Thermo Scientific para possibilitar a avaliação dos mesmos com o sistema Spark-DAT.

A configuração eletrônica do espectrômetro óptico foi modificada com a instalação do sistema para permitir a aquisição dos dados de intensidades após cada centelha individual. Os canais de 28 elementos químicos foram interligados a este sistema, dentre eles Al, Si, Mn, S, Mg, Ca e Ti.

Para cada característica mensurada (tipo, tamanho e número), o programa utilizou os algoritmos desenvolvidos pelo fabricante para coletar os sinais de cada elemento e gerar informações sobre as inclusões encontradas. Foram aplicados os algoritmos de contagem do número de inclusões por elemento e de compostos multielementares com até quatro espécies. Além disso, também foi utilizado o algoritmo que efetua o cálculo do tamanho das inclusões. As amostras foram mensuradas respeitando o limite da região demarcada previamente.

\section{RESULTADOS E DISCUSSÃO}

\subsection{Composição Química das Inclusões}

O exame das amostras $\left(A_{1}\right.$ a $\left.A_{9}\right)$ realizado por MEV/EDS indicou a predominância de inclusões contendo $\mathrm{Al}, \mathrm{O}, \mathrm{Ca}, \mathrm{S}$ e $\mathrm{Mg}$ como observado no espectro obtido da Figura 6. A avaliação destes componentes e de seu percentual em massa dado pela microanálise química por energia dispersiva revelou a presença de inclusões de alumina $\left(\mathrm{Al}_{2} \mathrm{O}_{3}\right)$, sulfeto de cálcio (CaS), e aluminatos de cálcio $\left(\mathrm{Al}_{2} \mathrm{O}_{3} \mathrm{x}\right.$.CaOy) puros ou combinados com óxidos de cálcio e de magnésio ( $\mathrm{CaO}$ e $\mathrm{MgO}$ ). Estas espécies são comumente encontradas em aços desoxidados ao alumínio, como é o caso do material estudado. Em algumas análises também foram observados traços de $\mathrm{Ti}, \mathrm{Nb}$ e $\mathrm{N}$, indicando a presença de nitretos destes metais.

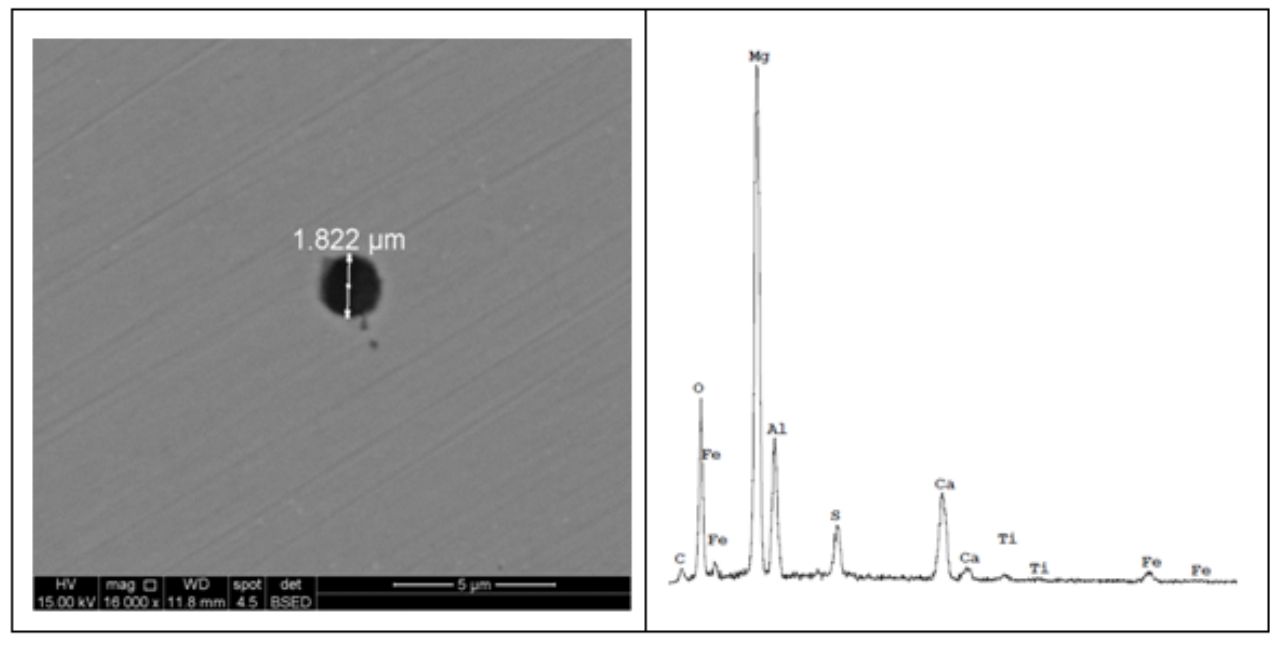

\begin{tabular}{|c|c|c|c|c|c|c|}
\hline \%O & \%Mg & \%Al & \%S & \%Ca & \%Ti & \%Fe \\
\hline 22,47 & 36,27 & 12,84 & 5,53 & 15,31 & 1,53 & 6,05 \\
\hline
\end{tabular}

Figura 6. Micrografia e espectro de EDS de uma inclusão encontrada na amostra $A_{3}$.

Nos diagramas de intensidade gerados para as amostras quando analisadas pelo Spark-DAT, foi possível verificar a presença de picos dos mesmos elementos

* Contribuição técnica ao $45^{\circ}$ Seminário de Aciaria - Internacional, 25 a 28 de maio de 2014, 
químicos encontrados predominantente pelo MEV/EDS, conforme mostrado pela Figura 7: Al, O, Ca, S, Mg. Este resultado demonstrou que existe uma coerência entre as duas técnicas de análise no que se refere à composição química das inclusões detectadas.

Ainda na Figura 7 pode-se observar que se combinados, estes diagramas confirmam a presença de inclusões de alumina, de sulfeto de cálcio, óxidos e aluminatos, como foi sugerido pela microscopia. Este fato pode ser observado pela ocorrência de picos de intensidade simultaneamente entre os elementos químicos destes compostos.

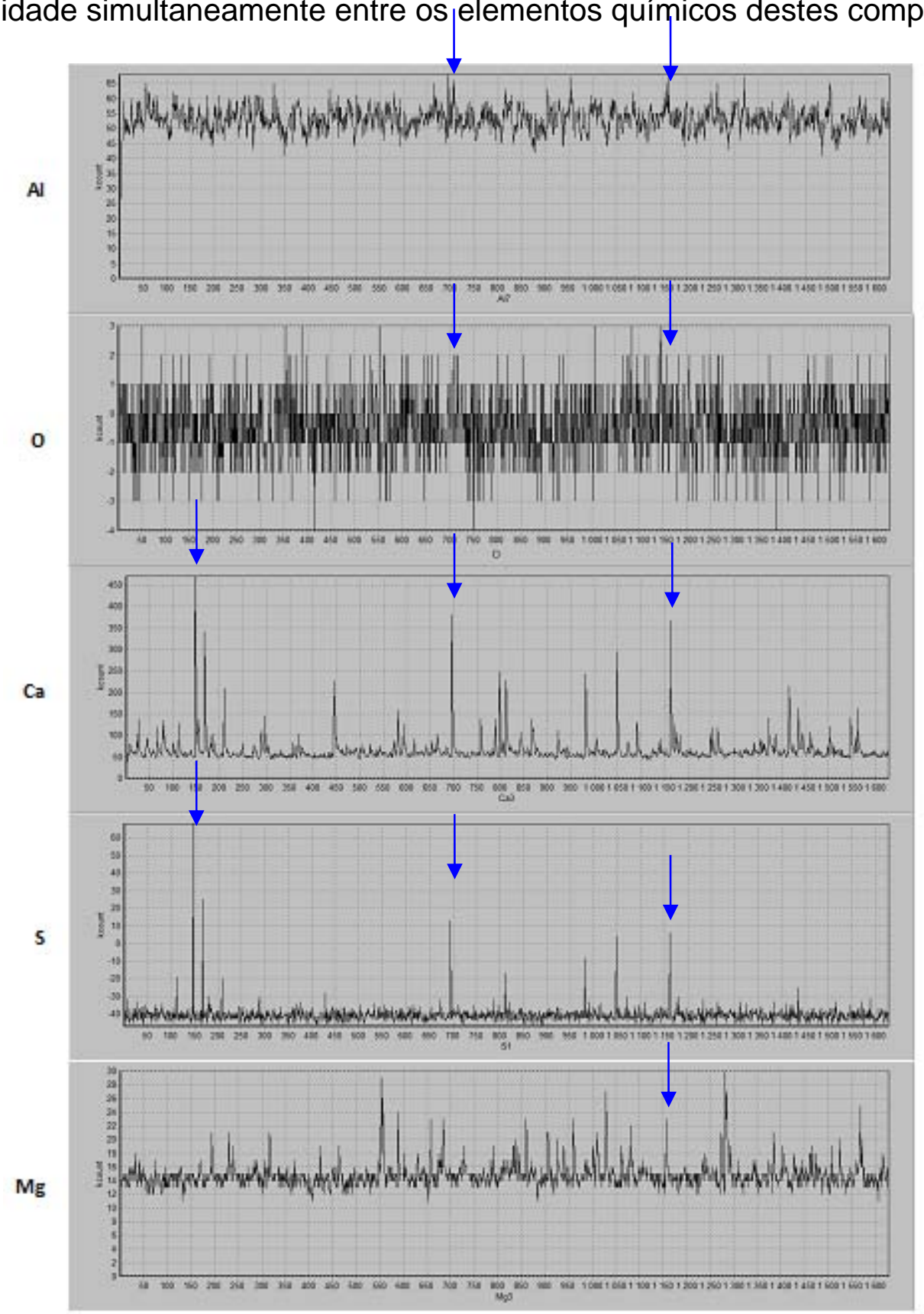

Figura 7. Diagramas de intensidades gerados pela amostra $A_{3}$.

\subsection{Tamanho das Inclusões}

Com o auxílio do microscópio eletrônico de varredura também foi possível mensurar o tamanho de cada inclusão avaliada, conforme mostrado na Figura 8. Todas as inclusões mensuradas situam-se na classe das microscópicas $(<100 \mu \mathrm{m})$ e

* Contribuição técnica ao $45^{\circ}$ Seminário de Aciaria - Internacional, 25 a 28 de maio de 2014, 
apresentam tamanhos dentro da faixa de $1 \mu \mathrm{m}$ a cerca de $10 \mu \mathrm{m}$. A maior parte delas possui diâmetro entre $1 \mu \mathrm{m}$ e $5 \mu \mathrm{m}$.

Resultado semelhante foi observado no exame das amostras com o Spark-DAT (ver também Figura 8), ou seja, foi encontrada uma distribuição de tamanhos deslocada para inclusões pequenas, de até aproximadamente $5 \mu \mathrm{m}$.
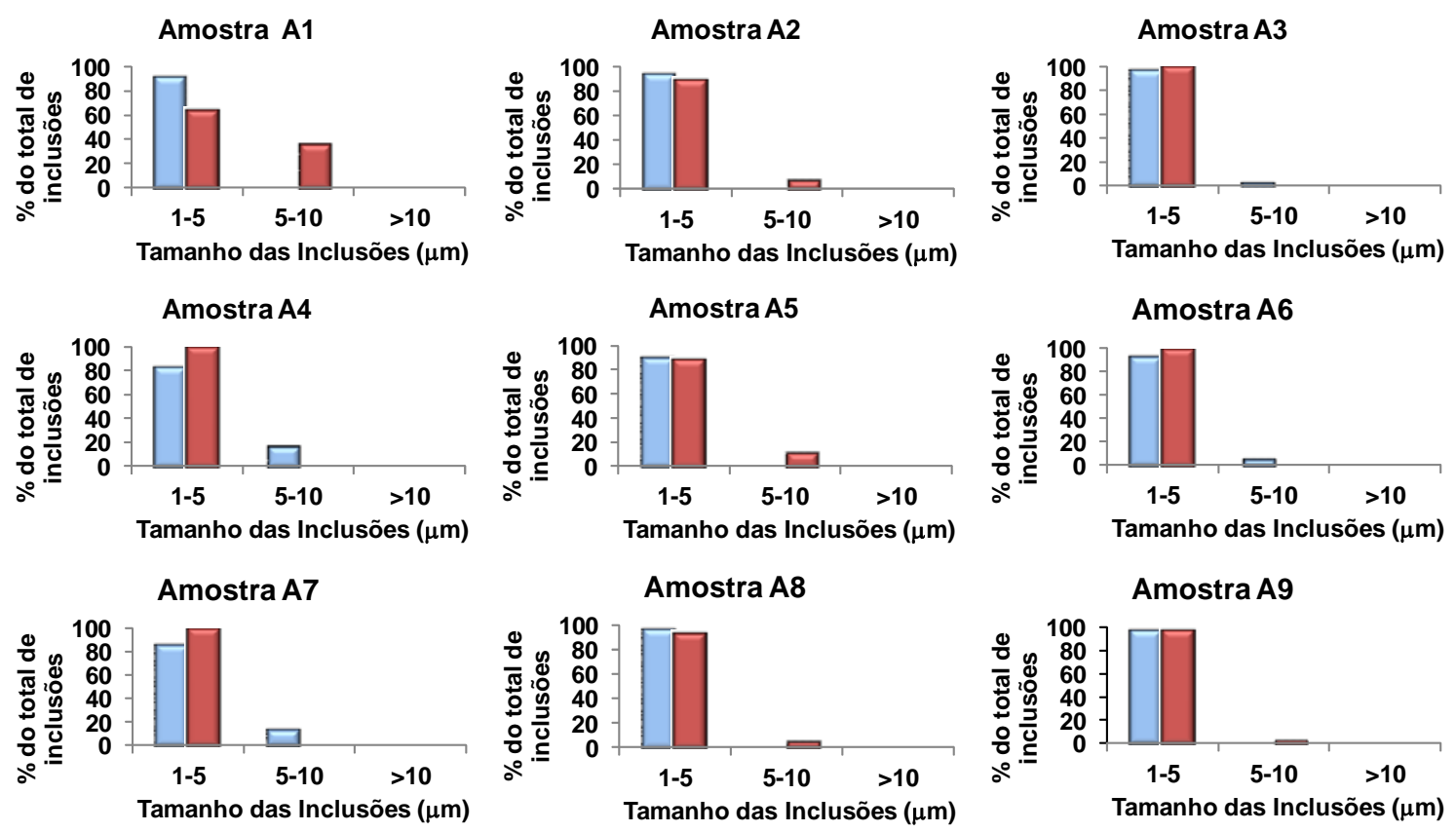

Legenda:

$\%$ MEV

$\%$ Spark-DAT

Figura 8. Distribuição de tamanho das inclusões com o MEV e o Spark-DAT.

Entretanto, quando os resultados são subdivididos em faixas menores de tamanho como aquelas demonstradas na figura 9, pode-se perceber que para a microscopia eletrônica, a maior parte das inclusões presentes possuem tamanhos entre 1 e $3 \mu \mathrm{m}$, e para o Spark-DAT entre 1 e $5 \mu \mathrm{m}$. Este comportamento do Spark-DAT que favorece a detecção de inclusões maiores que o MEV também foi observado em algumas pesquisas encontradas na literatura. Segundo Pande et al. [6], esse fato pode significar que duas ou mais inclusões muito pequenas podem estar sendo removidas em uma única centelha, dando a impressão de que foi encontrada uma inclusão de tamanho maior que o real.
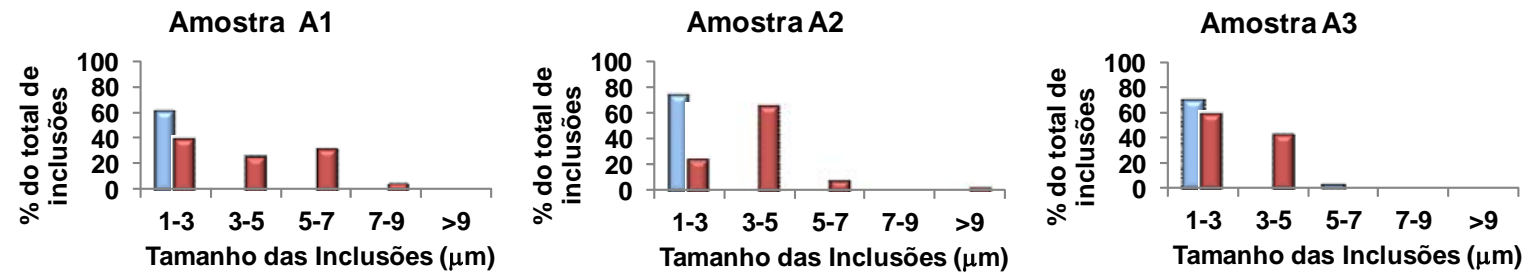

Legenda:

$\%$ MEV

$\%$ Spark-DAT

Figura 9. Distribuição de tamanho das inclusões estratificada.

* Contribuição técnica ao $45^{\circ}$ Seminário de Aciaria - Internacional, 25 a 28 de maio de 2014, Porto Alegre, RS, Brasil. 
Outra explicação pode estar no fato de o Spark-DAT ser um método de análise em volume, conforme mostra a Figura 10. Assim, como cada centelha remove uma pequena quantidade da amostra, a técnica pode detectar inclusões com um melhor alcance em profundidade.

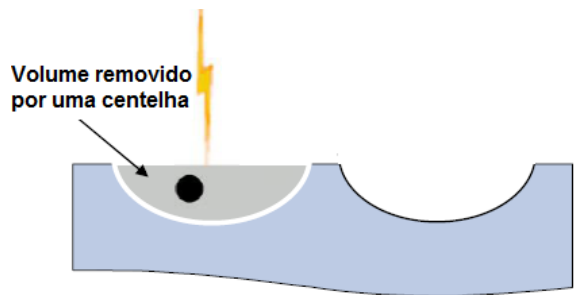

Figura 10. Análise em profundidade a partir da extração de um pequeno volume da amostra [4].

Segundo Böhlen [4], na microscopia, por ser uma técnica de análise superficial, as inclusões podem ser consideradas menores do que realmente são, caso o corte da amostra se dê em uma seção superficial (Figura 11).

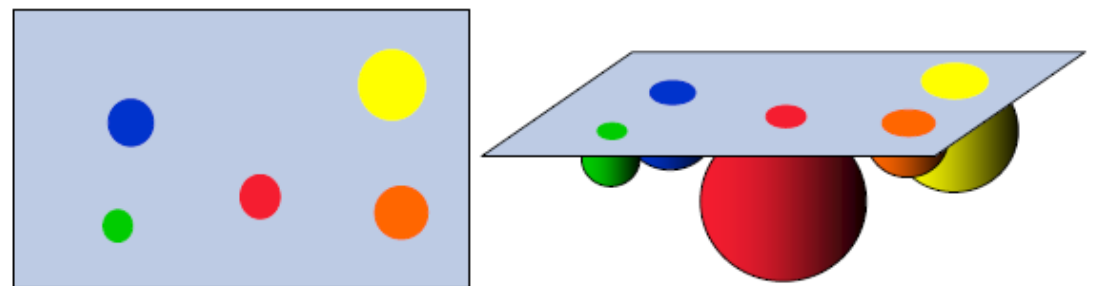

Figura 11. Inclusões relatadas como menores que seu tamanho real na microscopia [4].

Contudo, esta diferença encontrada entre as duas técnicas de medição não é significativa a ponto de inviabilizar a utilização do sistema para a aplicação na rotina de produção.

\subsection{Número de Inclusões Presentes}

Quanto ao número de inclusões detectadas, ao confrontar o Spark-DAT e a microscopia óptica na avaliação das amostras $A_{10}$ a $A_{12}$, verifica-se que as duas técnicas apresentaram resultados semelhantes como mostra a Tabela 1.

Tabela 1. Comparativo entre os resultados de microscopia óptica e do Spark-DAT

\begin{tabular}{|c|c|c|c|c|c|c|c|c|}
\hline \multirow{3}{*}{ Amostra } & \multicolumn{4}{|c|}{ Microscopia Óptica } & \multicolumn{4}{c|}{ Spark-DAT } \\
\cline { 2 - 8 } & Sulfeto & Alumina & Silicato & Óxido & \multirow{2}{*}{ Sulfeto } & Alumina & Silicato & Óxido \\
\cline { 2 - 8 } & \multicolumn{3}{|c|}{ (Série fina e nível 0,5 } & $1,0)$ & & & & \\
\hline $\mathbf{A}_{10}$ & 9 & 3 & 0 & 98 & 6 & 6 & 0 & 18 \\
\hline $\mathbf{A}_{11}$ & 7 & 6 & 0 & 94 & 5 & 3 & 0 & 15 \\
\hline $\mathbf{A}_{12}$ & 4 & 10 & 0 & 84 & 2 & 7 & 0 & 11 \\
\hline
\end{tabular}

Embora na microscopia óptica a norma de análise envolva a contagem de campos (áreas delimitadas) com inclusões e não do número de inclusões, foi possível observar nos dois métodos a presença de poucos sulfetos e aluminas e uma maior quantidade de óxidos.

A divergência identificada entre os resultados de óxidos possivelmente ocorreu devido à diferença entre os métodos de contagem e entre as áreas avaliadas pelas

* Contribuição técnica ao 450 Seminário de Aciaria - Internacional, 25 a 28 de maio de 2014, 
duas técnicas. Foi adotada uma área maior para a microscopia devido ao procedimento exigido pelo Método D (área mínima para análise de $160 \mathrm{~mm}^{2}$ ).

Para trabalhos futuros pode-se testar uma metodologia de contagem direta de inclusões no microscópio óptico no lugar da contagem de inclusões por campo. Assim, a comparação entre as duas técnicas possivelmente seria mais facilitada.

\section{CONCLUSÕES}

A partir dos resultados obtidos, é possível afirmar que o Spark-DAT é uma ferramenta capaz de efetuar com boa confiabilidade uma rápida avaliação da composição química, do tamanho e do número de inclusões presentes em amostras de aço. Esta caracterização pode ser realizada simultaneamente com a análise química do material, o que a torna ainda mais atrativa para aplicação na siderurgia.

A grande vantagem oferecida pelo método quando comparado com os convencionais é a elevada quantidade de informações fornecidas em pouco segundos, o que o torna bastante útil para diversas aplicações na área siderúrgica. Além disso, com uma única análise podem ser mensuradas centenas de inclusões, tarefa difícil para as demais técnicas atuais.

Os resultados deste comparativo mostraram coerência entre todas as metodologias utilizadas quanto à composição química das inclusões detectadas e seus respectivos tamanhos. No aço estudado foram detectadas inclusões contendo $\mathrm{Al}, \mathrm{O}$, $\mathrm{Ca}$, S e Mg que, no Spark-DAT, foram traduzidas em alumina $\left(\mathrm{Al}_{2} \mathrm{O}_{3}\right)$, sulfeto de cálcio (CaS) e aluminatos de cálcio $\left(\mathrm{Al}_{2} \mathrm{O}_{3} \mathrm{x}\right.$. CaOy) puros ou combinados.

Quanto ao tamanho, observou-se que o Spark-DAT apresentou uma tendência a informar resultados um pouco maiores que os produzidos pelo MEV, mas este desvio não inviabiliza a utilização do método, pois é pouco significativo. A técnica possui uma vantagem sobre a microscopia eletrônica, pois permite a análise da inclusão com uma maior profundidade e não somente em sua superfície. Desta forma, uma medida mais realista dos tamanhos das microinclusões pode ser obtida.

\section{Agradecimentos}

Os autores agradecem a Vallourec Tubos do Brasil e ao Departamento de Metalurgia da Universidade Federal de Minas Gerais pelo apoio fornecido ao longo deste trabalho.

\section{REFERÊNCIAS}

1 Kiessling R, Lange N. Non-metallic Inclusions in stell. London: The Metals Society; 1978.

2 Li K, Halász E, Böhlen JM. Ultra-fast quantitative analysis of non-metallic inclusions in steel. Londres: Millennium Steel; 2011. p.179-182.

3 Thermo Fischer Scientific. Spark-DAT Option: on-line single spark analysis: Application Note: 41232. Ecublens, Suíça; 2007. p.4.

4 Böhlen JM. Latest developments in optical emission spectroscopy. In: Meeting Spark-dat \& Automation; 2011. Belo Horizonte: Thermo Fisher Scientific; 2011. p.71.

5 ASTM International. ASTM E45-05 - Standard test methods for determining the inclusion content of steel. West Conshohocken: American Society for Testing and Materials; 2005. p.19.

6 Pande MM, Guo M, Dumarey R, Devisscher S, Blanpain B. Determination of steel cleanliness in ultra low carbon steel by pulse discrimination analysis optical emission spectroscopy technique. ISIJ International. 2001;51(11):1778-87.

* Contribuição técnica ao $45^{\circ}$ Seminário de Aciaria - Internacional, 25 a 28 de maio de 2014, 